This is an electronic reprint of the original article. This reprint may differ from the original in pagination and typographic detail.

Author(s): Brambilla, Nora; Escobedo Espinosa, Miguel; Soto, Joan; Vairo, Antonio

Title: Quarkonium suppression in heavy-ion collisions: An open quantum system approach

Year: $\quad 2017$

Version:

Please cite the original version:

Brambilla, N., Escobedo Espinosa, M., Soto, J., \& Vairo, A. (2017). Quarkonium suppression in heavy-ion collisions: An open quantum system approach. Physical Review D, 96(3), Article 034021. https://doi.org/10.1103/PhysRevD.96.034021

All material supplied via JYX is protected by copyright and other intellectual property rights, and duplication or sale of all or part of any of the repository collections is not permitted, except that material may be duplicated by you for your research use or educational purposes in electronic or print form. You must obtain permission for any other use. Electronic or print copies may not be offered, whether for sale or otherwise to anyone who is not an authorised user. 
PHYSICAL REVIEW D 96, 034021 (2017)

\title{
Quarkonium suppression in heavy-ion collisions: An open quantum system approach
}

\author{
Nora Brambilla \\ Physik Department, Technische Universität München, D-85748 Garching, Germany \\ and Institute for Advanced Study, Technische Universität München, Lichtenbergstrasse 2 a, \\ D-85748 Garching, Germany \\ Miguel A. Escobedo \\ Department of Physics, P.O. Box 35, FI-40014 University of Jyväskylä, Finland \\ and Institut de Physique Théorique, Université Paris Saclay, CNRS, CEA, F-91191 Gif-sur-Yvette, France \\ Joan Soto \\ Departament de Física Quàntica i Astrofísica and Institut de Ciències del Cosmos, \\ Universitat de Barcelona, Martí i Franquès 1, 08028 Barcelona, Catalonia, Spain \\ Antonio Vairo \\ Physik Department, Technische Universität München, D-85748 Garching, Germany
} (Received 30 December 2016; published 25 August 2017)

\begin{abstract}
We address the evolution of heavy-quarkonium states in an expanding quark-gluon plasma by implementing effective field theory techniques in the framework of open quantum systems. In this setting we compute the nuclear modification factors for quarkonia that are $S$-wave Coulombic bound states in a strongly coupled quark-gluon plasma. The calculation is performed at an accuracy that is leading order in the heavy-quark density expansion and next-to-leading order in the multipole expansion. The quarkonium density-matrix evolution equations can be written in the Lindblad form, and, hence, they account for both dissociation and recombination. Thermal mass shifts, thermal widths and the Lindblad equation itself depend on only two nonperturbative parameters: the heavy-quark momentum diffusion coefficient and its dispersive counterpart. Finally, by numerically solving the Lindblad equation, we provide results for the $\Upsilon(1 S)$ and $\Upsilon(2 S)$ suppression.
\end{abstract}

DOI: 10.1103/PhysRevD.96.034021

\section{INTRODUCTION}

Quarkonium suppression has long since been suggested as a hard probe for the quark-gluon plasma formed in highenergy heavy-ion collisions [1]. Experimentally, quarkonium provides a potentially clean signal through dilepton decays [2]. Theoretically, it allows the use of nonrelativistic effective field theories to factorize high-energy contributions from low-energy ones. The latter can be eventually computed by lattice QCD [3-5]. Nevertheless, many processes contribute to the final heavy-quarkonium observables, among these the hydrodynamic evolution of the plasma, and quarkonium production, dissociation and regeneration in the different medium conditions.

The problem may be simplified if one considers the quarkonium ground state and particularly the bottomonium $1 S$ states. (To a lesser extent the same may apply to the charmonium ground state and to the bottomonium $2 S$ states.) In this case not only can one argue that the mass of the heavy quark, $m$, is the largest scale of the problem, which qualifies the system as nonrelativistic, but also that the typical momentum transfer between the heavy quarks is the next relevant scale for a certain range of temperatures, which qualifies the system as Coulombic. The Bohr radius of such a system, $a_{0} \sim 1 /\left(m \alpha_{s}\right)$ (the inverse of the typical momentum transfer), is the scale at which the strong coupling is computed in the potential. The Coulomb potential of a quark-antiquark pair in a color-singlet configuration reads $V_{s}(r)=-C_{F} \alpha_{s} / r$, and in a color-octet configuration $V_{o}(r)=\alpha_{s} /\left(2 N_{c} r\right) ; N_{c}=3$ is the number of colors and $C_{F}=\left(N_{c}^{2}-1\right) /\left(2 N_{c}\right)=4 / 3$ is the Casimir of the fundamental representation of $\mathrm{SU}(3)$.

At least two mechanisms of quarkonium decay in a medium have been identified over the years: gluodissociation $[6,7]$ and dissociation via parton scattering $[8,9]$. Both dissociation mechanisms have been studied in an effective field theory framework applied to Coulombic heavy quarkantiquark states in a weakly coupled plasma in [10-15]. An extensive phenomenological analysis of bottomonium suppression can be found in [16-18].

In a weakly coupled plasma one assumes the hierarchy $\pi T \gg m_{D}$, where $T$ is the temperature and $m_{D} \sim g T$ the Debye mass of the plasma (the factor $\pi$ is a remnant of the Matsubara frequencies). The theoretical advantage from this situation is that one may use perturbation theory as a computational tool. It is uncertain, however, if a weakly 
coupled plasma is what best describes the medium formed in heavy-ion collisions at the LHC. A more conservative approach consists in assuming that the plasma is strongly coupled, i.e., $\pi T \sim m_{D}$. This is the situation that we will analyze in the following, where we will assume the hierarchy of scales:

$$
m \gg \frac{1}{a_{0}} \sim m \alpha_{s} \gg \pi T \sim m_{D} \gg \text { any other scale. }
$$

The other scales include the binding energy and $\Lambda_{\mathrm{QCD}}$, whose relative size is not specified. We will compute the quarkonium thermal decay width and mass shift, write and solve the heavy quark-antiquark evolution equations, and finally evaluate the bottomonium nuclear modification factor.

Bottomonium suppression has been measured by CMS at $2.76 \mathrm{TeV}$ [19]. Analyses for LHC data at $5.02 \mathrm{TeV}$ are under way (see, e.g., [20]). Recently also the STAR experiment has considered bottomonium suppression data at the much lower energy of $193 \mathrm{GeV}$ improving on previous studies by the PHENIX collaboration [21].

\section{QUARKONIUM DECAY WIDTH AND MASS SHIFT IN A STRONGLY COUPLED PLASMA}

Under condition (1), the effective field theory suited to describe heavy quark-antiquark pairs at an energy scale lower than $m \alpha_{s}$ but larger than the thermal scales, is potential nonrelativistic QCD (pNRQCD) [22-24]. According to the hierarchy of energy scales, pNRQCD may be computed setting to zero the temperature and any other scale lower than $m \alpha_{s}$ [10]. The remaining scales provide nonperturbative contributions: contributions from thermal scales have to be resummed to all orders because they are induced by a strongly coupled plasma, but also contributions scaling with the binding energy of the system may be nonperturbative if the binding energy is not larger than $\Lambda_{\mathrm{QCD}}$.

The Lagrangian of pNRQCD at next-to-leading order in the multipole expansion reads

$$
\begin{aligned}
\mathcal{L}_{\text {pNRQCD }}= & \int d^{3} r \operatorname{Tr}\left[\mathrm{S}^{\dagger}\left(i \partial_{0}-h_{s}\right) \mathrm{S}+\mathrm{O}^{\dagger}\left(i D_{0}-h_{o}\right) \mathrm{O}\right] \\
& +\operatorname{Tr}\left[\left(\mathrm{O}^{\dagger} \mathbf{r} \cdot g \mathbf{E S}+\text { H.c. }\right)+\frac{1}{2}\left(\mathrm{O}^{\dagger} \mathbf{r} \cdot g \mathbf{E O}+\text { c.c. }\right)\right] \\
& +\mathcal{L}_{\text {light }},
\end{aligned}
$$

where $r$ is the distance between the heavy quark and the antiquark (the above Lagrangian is accurate up to order $r$ ), $\mathrm{S}=S \mathbb{1}_{c} / \sqrt{N_{c}}$ and $\mathrm{O}=\sqrt{2} O^{a} T^{a}$ stand for the heavy quarkantiquark fields in a color-singlet and a color-octet configuration respectively, $h_{s}=\mathbf{p}^{2} / m+V_{s}$ is the color-singlet Hamiltonian, $h_{o}=\mathbf{p}^{2} / m+V_{o}$ is the color-octet Hamiltonian $\left(\mathbf{p}=-i \boldsymbol{\nabla}_{r}\right), \mathbf{E}$ is the chromoelectric field, $g$ the strong coupling and H.c. and c.c. stand for Hermitian conjugate and charge conjugate, respectively. The term $\mathcal{L}_{\text {light }}$ denotes the QCD Lagrangian with light quarks only. The covariant derivative acting on the octet field $\mathrm{O}$ in (2) can be eliminated by means of suitable field redefinitions: $\mathrm{O} \rightarrow \Omega \mathrm{O} \Omega^{\dagger}$ and $\mathbf{E} \rightarrow \Omega \mathbf{E} \Omega^{\dagger}$, where $\Omega=$ $\mathrm{P} \exp \left[-i g \int_{-\infty}^{t} d s A_{0}(s, \mathbf{R})\right]$ and $\mathbf{R}$ is the center of mass coordinate. We use these field redefinitions when deriving the evolution equations in the next section.

At order $r$ in the multipole expansion quark-antiquark pairs interact with the medium through chromoelectricdipole interactions. In (2) we have neglected radiative corrections to the Wilson coefficients of these terms, since they are beyond our accuracy. According to (1) the thermodynamical scales are much larger than the binding energy. Hence, the latter can be neglected in the colorsinglet self-energy, which reads

$$
\Sigma_{s}(t)=\frac{g^{2}}{6 N_{c}} r^{2} \int_{t_{0}}^{t} d t_{2}\left\langle E^{a, i}(t, \mathbf{0}) E^{a, i}\left(t_{2}, \mathbf{0}\right)\right\rangle,
$$

where $\langle\cdots\rangle$ stands for the thermal average. The time $t_{0}$ is the formation time of the quark-gluon plasma, i.e., the initial time for the evolution of the heavy quark-antiquark pairs in the medium.

In the following, we will assume that $t-t_{0}$ is larger than any other time scale of the system and that the evolution of the temperature is quasistatic: $1 / T \times d T / d t \sim 1 / t \lesssim$ bindingenergy. As a consequence of these two assumptions we can approximate $\int_{t_{0}}^{t} d t_{2}\left\langle E^{a, i}(t, \mathbf{0}) E^{a, i}\left(t_{2}, \mathbf{0}\right)\right\rangle \approx$ $\int_{-\infty}^{+\infty} d s\left\langle\mathrm{~T} E^{a, i}(s, \mathbf{0}) E^{a, i}(0, \mathbf{0})\right\rangle / 2$. The last correlator is the time-ordered one. It may be computed as if the medium was in thermal equilibrium at a slowly varying temperature $T$.

The leading contribution to the thermal decay width is given by the imaginary part of the color-singlet self-energy. Under the above assumptions, it reads for $1 S$ states

$$
\Gamma=-2\left\langle\operatorname{Im}\left(-i \Sigma_{s}\right)\right\rangle=3 a_{0}^{2} \kappa
$$

The heavy-quark momentum diffusion coefficient, $\kappa$, is defined as $[25,26]$

$$
\kappa=\frac{g^{2}}{6 N_{c}} \operatorname{Re} \int_{-\infty}^{+\infty} d s\left\langle\mathrm{~T} E^{a, i}(s, \mathbf{0}) E^{a, i}(0, \mathbf{0})\right\rangle .
$$

A recent lattice determination of $\kappa$ found [27]

$$
1.8 \lesssim \frac{\kappa}{T^{3}} \lesssim 3.4
$$

This estimate has been obtained from a pure SU(3) plasma at a temperature of about $1.5 T_{c}$. With this value of $\kappa$, $m_{b}=4.8 \mathrm{GeV}$ and $1 / a_{0}=1.334 \mathrm{GeV}$ that follows from the self-consistency equation $1 / a_{0}=m_{b} C_{F} \alpha_{s}\left(1 / a_{0}\right) / 2$, we obtain for the $\Upsilon(1 S): 3.0 \mathrm{GeV}^{-2} T^{3} \lesssim \Gamma_{\Upsilon(1 S)} \lesssim$ $5.7 \mathrm{GeV}^{-2} T^{3}$. This provides a thermal width around 
$100 \mathrm{MeV}$ for temperatures of about $300 \mathrm{MeV}$. We recall that the crossover temperature to the quark-gluon plasma, $T_{c}$, as measured by lattice QCD is about $150 \mathrm{MeV}$ [28-30].

The leading contribution to the quarkonium mass shift is given by the real part of the color-singlet self-energy. For $1 S$ states it reads

$$
\delta m=\left\langle\operatorname{Re}\left(-i \Sigma_{s}\right)\right\rangle=\frac{3}{2} a_{0}^{2} \gamma,
$$

where

$$
\gamma=\frac{g^{2}}{6 N_{c}} \operatorname{Im} \int_{-\infty}^{+\infty} d s\left\langle\mathrm{~T} E^{a, i}(s, \mathbf{0}) E^{a, i}(0, \mathbf{0})\right\rangle
$$

So far the only estimate that we have for $\gamma$ is the perturbative calculation done at leading order in [10]:

$$
\gamma=-2 \zeta(3) C_{F}\left(\frac{4}{3} N_{c}+n_{f}\right) \alpha_{s}^{2} T^{3}
$$

where $n_{f}$ is the number of light quarks. In the case of a strongly coupled plasma, however, there is no reason to believe that perturbation theory provides a good approximation of $\gamma$. For example, the leading-order estimate of $\kappa$ is far off the nonperturbative result [31].

\section{EVOLUTION EQUATIONS}

The yield of quarkonium $n S$ states in heavy-ion collisions normalized with respect to the yield in $p p$ collisions, as measured from dilepton decays, is called the quarkonium nuclear modification factor, $R_{A A}(n S)$. It can be expressed as the density of (color singlet) $n S$ states in heavy-ion collisions normalized with respect to the same quantity in $p p$ collisions $[2,32]$. The density of color-singlet heavy quark-antiquark states, $\rho_{s}$, and color-octet ones, $\rho_{o}^{b a}=\rho_{o} \delta^{a b} /\left(N_{c}^{2}-1\right)$, may be expressed in the close-time-path formalism as a singlet and octet propagator, respectively, that propagate from the upper branch (labeled 1) to the lower branch (labeled 2) of the time path [33]: $\left\langle\mathbf{r}^{\prime}\left|\rho_{s}(t ; t)\right| \mathbf{r}\right\rangle=\operatorname{Tr}\left\{\rho S^{\dagger}(t, \mathbf{r}, \mathbf{R}) S\left(t, \mathbf{r}^{\prime}, \mathbf{R}\right)\right\}=$ $\left\langle S_{1}\left(t, \mathbf{r}^{\prime}, \mathbf{R}\right) S_{2}^{\dagger}(t, \mathbf{r}, \mathbf{R})\right\rangle, \quad\left\langle\mathbf{r}^{\prime}\left|\rho_{o}(t ; t)\right| \mathbf{r}\right\rangle \delta^{a b} /\left(N_{c}^{2}-1\right)=$ $\operatorname{Tr}\left\{\rho O^{a \dagger}(t, \mathbf{r}, \mathbf{R}) O^{b}\left(t, \mathbf{r}^{\prime}, \mathbf{R}\right)\right\}=\left\langle O_{1}^{b}\left(t, \mathbf{r}^{\prime}, \mathbf{R}\right) O_{2}^{a^{\dagger}}(t, \mathbf{r}, \mathbf{R})\right\rangle$. We have assumed that the heavy quarks comove with the medium, so that we do not need to consider the center of mass motion.

The evolution equations for the singlet and octet densities may be computed, therefore, by considering selfenergy contributions similar to the one considered in the previous section, but now describing the evolution of the $\left\langle S_{1} S_{2}^{\dagger}\right\rangle$ and $\left\langle O_{1}^{b} O_{2}^{a \dagger}\right\rangle$ propagators. Keeping only terms linear in the heavy-quark densities and resumming selfenergy contributions by means of a Schwinger-Dyson equation, we obtain the evolution equations:

$$
\begin{aligned}
\frac{d \rho_{s}(t ; t)}{d t}= & -i\left[h_{s}, \rho_{s}(t ; t)\right]-\Sigma_{s}(t) \rho_{s}(t ; t)-\rho_{s}(t ; t) \Sigma_{s}^{\dagger}(t) \\
& +\Xi_{s o}\left(\rho_{o}(t ; t), t\right), \\
\frac{d \rho_{o}(t ; t)}{d t}= & -i\left[h_{o}, \rho_{o}(t ; t)\right]-\Sigma_{o}(t) \rho_{o}(t ; t)-\rho_{o}(t ; t) \Sigma_{o}^{\dagger}(t) \\
& +\Xi_{o s}\left(\rho_{s}(t ; t), t\right)+\Xi_{o o}\left(\rho_{o}(t ; t), t\right),
\end{aligned}
$$

where, assuming the hierarchy of energy scales (1) that allows to neglect energy-dependent exponentials, we have

$$
\begin{gathered}
\Sigma_{s}(t)=\frac{r^{2}}{2}[\kappa(t)+i \gamma(t)], \\
\Sigma_{o}(t)=\frac{N_{c}^{2}-2}{2\left(N_{c}^{2}-1\right)} \frac{r^{2}}{2}[\kappa(t)+i \gamma(t)], \\
\Xi_{s o}\left(\rho_{o}, t\right)=\frac{1}{N_{c}^{2}-1} r^{i} \rho_{o} r^{i} \kappa(t), \\
\Xi_{o s}\left(\rho_{s}, t\right)=r^{i} \rho_{s} r^{i} \kappa(t), \\
\Xi_{o o}\left(\rho_{o}, t\right)=\frac{N_{c}^{2}-4}{2\left(N_{c}^{2}-1\right)} r^{i} \rho_{o} r^{i} \kappa(t) .
\end{gathered}
$$

The equations show the coupled evolution of the singlet and octet densities. Their interpretation is straightforward: the function $\Xi_{s o}$ accounts for the production (or regeneration) of singlets through the decay of octets, while the functions $\Xi_{o s}$ and $\Xi_{o o}$ account for the production of octets through the decays of singlets and octets, respectively. These two octet production mechanisms can be traced back to the two different sets of chromoelectric dipole operators in the pNRQCD Lagrangian (2).

With the above functions, we can rewrite the evolution Eqs. (10) and (11) in the Lindblad form [34,35]:

$$
\frac{d \rho}{d t}=-i[H, \rho]+\sum_{n}\left(C_{n} \rho C_{n}^{\dagger}-\frac{1}{2}\left\{C_{n}^{\dagger} C_{n}, \rho\right\}\right),
$$

where $H$ is a Hermitian operator and the operators $C_{n}$ are called collapse operators. In our case, the matrix $\rho$ is

$$
\rho=\left(\begin{array}{cc}
\rho_{s} & 0 \\
0 & \rho_{o}
\end{array}\right)
$$

the operator $H$ is

$$
H=\left(\begin{array}{cc}
h_{s} & 0 \\
0 & h_{o}
\end{array}\right)+\frac{r^{2}}{2} \gamma(t)\left(\begin{array}{cc}
1 & 0 \\
0 & \frac{N_{c}^{2}-2}{2\left(N_{c}^{2}-1\right)}
\end{array}\right),
$$

and we need six collapse operators, 
TABLE I. Initial temperature of the fireball, $T_{0}$, for different centrality bins $(10 \%$ centrality means that the $10 \%$ most central collisions have been selected) computed by means of the Glauber model from the mean impact parameters, $\langle b\rangle$, taken from [39].

\begin{tabular}{lcc}
\hline \hline Centrality $(\%)$ & $\langle b\rangle(\mathrm{fm})$ & $T_{0}(\mathrm{MeV})$ \\
\hline $0-10$ & 3.4 & 471 \\
$10-20$ & 6.0 & 461 \\
$20-30$ & 7.8 & 449 \\
$30-50$ & 9.9 & 425 \\
$50-100$ & 13.6 & 304 \\
\hline \hline
\end{tabular}

$$
\begin{aligned}
& C_{i}^{0}=\sqrt{\frac{\kappa(t)}{N_{c}^{2}-1}} r^{i}\left(\begin{array}{cc}
0 & 1 \\
\sqrt{N_{c}^{2}-1} & 0
\end{array}\right), \\
& C_{i}^{1}=\sqrt{\frac{\left(N_{c}^{2}-4\right) \kappa(t)}{2\left(N_{c}^{2}-1\right)}} r^{i}\left(\begin{array}{ll}
0 & 0 \\
0 & 1
\end{array}\right) .
\end{aligned}
$$

The Lindblad equation has been studied in relation with quarkonium in a quark-gluon plasma also in [36].

\section{BOTTOMONIUM SUPPRESSION}

We assume that the temperature of the quark-gluon plasma evolves slowly according to

$$
T=T_{0}\left(\frac{t_{0}}{t}\right)^{v_{s}^{2}},
$$

where $T_{0}$ is the initial temperature and $v_{s}$ is the velocity of sound in the medium [37]. In a deconfined plasma at a very high temperature $v_{s}^{2}=1 / 3$. As values of $T_{0}$ and $t_{0}$ for central collisions at the LHC we take $T_{0}=475 \mathrm{MeV}$ and $t_{0}=0.6 \mathrm{fm}[38]$.

We study collisions two different centrality classes in this work, $30 \%-50 \%$ and $50 \%-100 \%$. The reason is that it is for

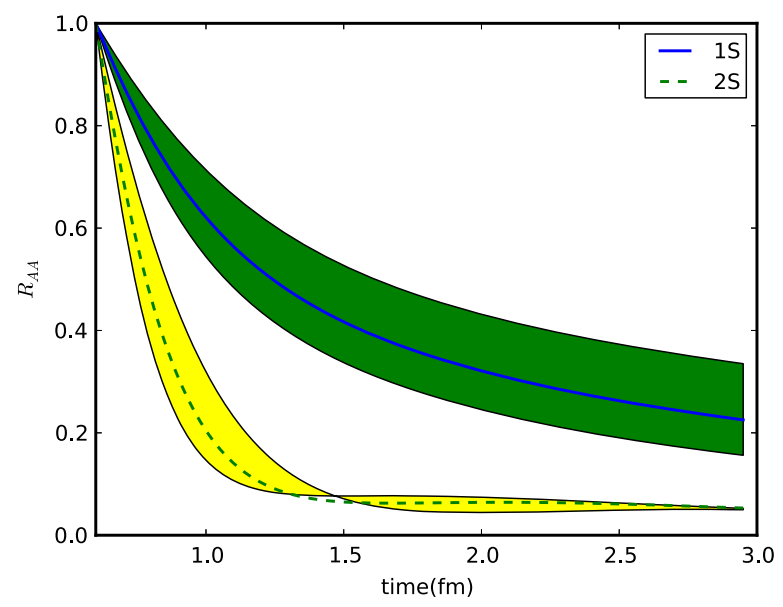

FIG. 1. Time evolution of $R_{A A}$ for $\kappa / T^{3}$ in the range (6), $\gamma=0$ and $\delta=1$, for $30 \%-50 \%$ centrality.

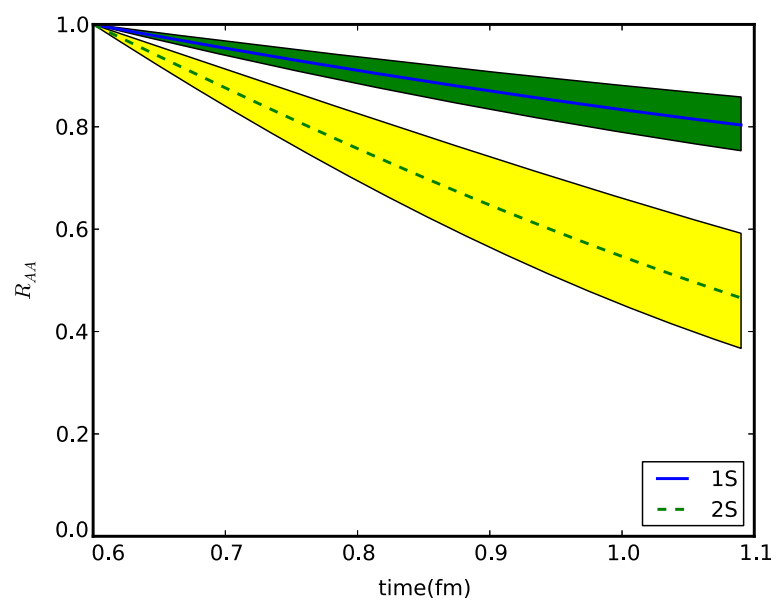

FIG. 2. Time evolution of $R_{A A}$ for $\kappa / T^{3}$ in the range (6), $\gamma=0$ and $\delta=1$, for $50 \%-100 \%$ centrality.

these classes where we believe that the hierarchy of Eq. (1) is more likely to be fulfilled. For a plasma that is homogeneous and isotropic the only effect that a difference in centrality produces is a change in the initial value of the energy density and hence in $T_{0}$. The values of $T_{0}$ for different centralities and mean impact parameters that we use are listed in Table I.

According to (22) and Table I, if the evolution starts at $t_{0}=0.6 \mathrm{fm}$, the fireball cools down to about $250 \mathrm{MeV}$ at about $4 \mathrm{fm}$ for the most central collisions and at about $1.1 \mathrm{fm}$ for the most peripheral ones. A temperature of about $250 \mathrm{MeV}$ is the smallest temperature, still larger than $T_{c}$, where we expect the hierarchy (1) to be safely realized for the bottomonium lower states. The outcome of the evolution equations depends on the initial condition. Both the singlet and the octet are initialized in a Dirac-delta state as they are assumed to be produced by a hard (local) process. We call $\delta / \alpha_{s}\left(m_{b}\right)$ the initial fraction of octets with respect to singlets (octet production is $1 / \alpha_{s}$ enhanced with respect to singlet production [40]). In the bottomonium case, the time evolutions of $R_{A A}$ for 30\%-50\% centrality and 50\%$100 \%$ centrality are shown in Figs. 1 and 2, respectively. Note that in Fig. 1 the $R_{A A}$ for the $2 S$ state becomes insensitive to $\kappa$ at large times, an indication that it reaches a steady state before the quark-gluon plasma vanishes.

We have taken $\kappa / T^{3}$ in the range (6), while we have set $\gamma=0$ and $\delta=1$. The choice of $\gamma$ is arbitrary, since a nonperturbative determination of this parameter is missing. The CMS results of [19] prefer lower values of $\gamma$, which is

TABLE II. Results for $R_{A A}(1 S)$ and $R_{A A}(2 S)$ for $\kappa / T^{3}$ in the range (6), $\gamma=0$ and $\delta=1$ in the bottomonium case.

\begin{tabular}{lccc}
\hline \hline \multicolumn{2}{c}{$30 \%-50 \%$} & centrality & \multicolumn{2}{c}{$50 \%-100 \%$ centrality } \\
\hline$R_{A A}(1 S)$ & $\frac{R_{A A}(2 S)}{R_{A A}(1 S)}$ & $R_{A A}(1 S)$ & $\frac{R_{A A}(2 S)}{R_{A A}(1 S)}$ \\
$0.23_{-0.07}^{+0.10}$ & $0.24 \pm 0.09$ & $0.80 \pm 0.05$ & $0.59 \pm 0.10$ \\
\hline \hline
\end{tabular}




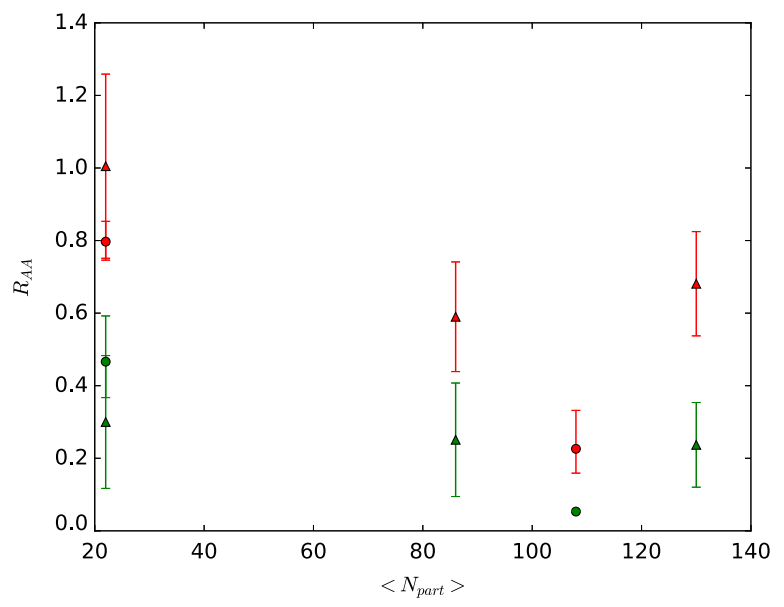

FIG. 3. $R_{A A}$ as obtained from Table II (dots) compared with the CMS data of [19] (triangles). Upper (red) entries refer to the $\Upsilon(1 S)$, lower (green) entries to the $\Upsilon(2 S)$.

the rational for our choice of $\gamma=0$. The choice $\delta=1$ assumes the initial ratio of octets over singlets to be just $1 / \alpha_{s}\left(m_{b}\right)$. The precise value of $\delta$ is not so important as the result is rather insensitive to it.

Our results are summarized in Table II and compared in Fig. 3 with the CMS results of [19]. Note that the results on this table are corrected for feed-down effects, which is an after freeze-out effect, using the method of [18] with the updated feed-down fractions of [17]. A more recent set of data is in [41]. An analysis that appeared after this work was completed and made public is in [42].

\section{CONCLUSIONS}

In this paper, we have considered heavy quarkonium in heavy-ion collision experiments as an open quantum system, and derived the evolution equations for the singlet and octet density matrices in (10) and (11) under the assumption that pNRQCD (2) is applicable, which is our main result. For the case of a strongly coupled plasma under the conditions (1), the equations depend only on two nonperturbative parameters, the heavy-quark momentum diffusion coefficient $\kappa$ and the parameter $\gamma$ respectively defined in (5) and (8), and, furthermore, can be written in the Lindblad form (17).

We have solved numerically the equations for the case of bottomonium, taking the initial conditions according to NRQCD production in vacuum and the evolution of the plasma according to Bjorken's model. We obtain the nuclear modification factors $R_{A A}(1 S)$ and $R_{A A}(2 S)$ (see
Table II). The output depends crucially on the parameters $\kappa$ and $\gamma$. While $\kappa$ has at least been computed in quenched lattice QCD for temperatures close to $T_{c}, \gamma$ has not, and, therefore, it remains as one of the main uncertainties in the determination of $R_{A A}$.

A detailed derivation of the results presented here as well as an analysis of the evolution equations for the case of a weakly coupled quark-gluon plasma will be presented in a forthcoming publication [32].

Recently, several works have addressed the computation of quarkonium suppression taking into account the quantum evolution, the imaginary part of the potential and the conservation of the number of heavy particles $[43,44]$, as it is also the case in this paper. However, an important difference is that in this work we have taken into account both color structures of the quark-antiquark pair, the singlet and the octet. This distinguishes it from the Abelian case and, as we have shown, has important phenomenological consequences. Finally, we emphasize that we have kept the full quantum-mechanical nature of the quarkonium during the whole evolution. To be definite we have presented results for Bjorken's model of hydrodynamic evolution and for a particular initial condition. Clearly this is not a limitation of the formalism, which allows any hydrodynamic evolution of the medium to be incorporated and the initial conditions to be tuned to account for preequilibrium states like Glasma (for a recent review see, e.g., [45]).

\section{ACKNOWLEDGMENTS}

N. B. and A. V. thank Torsten Dahms for many communications. The work of N. B. and A. V. was supported by the Bundesministerium für Bildung und Forschung (BMBF) through the "Verbundprojekt 05P2015-ALICE at High Rate (BMBF-FSP 202) GEM-TPC Upgrade and Field theory based investigations of ALICE physics" under Grant No. 05P15WOCA1 and by the DFG cluster of excellence "Origin and structure of the universe" [46]. The work of M. A.E. was supported by the European Research Council under the Advanced Investigator Grant No. ERC-AD267258 and by the Academy of Finland, Project No. 303756. J. S. thanks N. B. and A. V. for hospitality at TUM. He has been supported by the CPAN CSD2007-00042 Consolider-Ingenio 2010 program, and the FPA2010-16963, FPA2013-43425-P and FPA2013-4657 projects (Spain), and the 2014-SGR-104 grant (Catalonia).
[1] T. Matsui and H. Satz, $J / \psi$ suppression by quarkgluon plasma formation, Phys. Lett. B 178, 416 (1986).
[2] L. D. McLerran and T. Toimela, Photon and dilepton emission from the quark-gluon plasma: Some general considerations, Phys. Rev. D 31, 545 (1985). 
[3] N. Brambilla et al., Heavy quarkonium physics, arXiv: hep-ph/0412158.

[4] N. Brambilla et al., Heavy quarkonium: Progress, puzzles, and opportunities, Eur. Phys. J. C 71, 1534 (2011).

[5] N. Brambilla et al., QCD and strongly coupled gauge theories: Challenges and perspectives, Eur. Phys. J. C 74, 2981 (2014).

[6] D. Kharzeev and H. Satz, Quarkonium interactions in hadronic matter, Phys. Lett. B 334, 155 (1994).

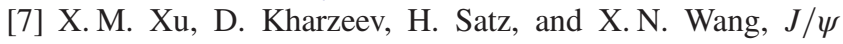
suppression in an equilibrating parton plasma, Phys. Rev. C 53, 3051 (1996).

[8] L. Grandchamp and R. Rapp, Thermal versus direct $J / \psi$ production in ultrarelativistic heavy ion collisions, Phys. Lett. B 523, 60 (2001).

[9] L. Grandchamp and R. Rapp, Charmonium suppression and regeneration from SPS to RHIC, Nucl. Phys. A709, 415 (2002).

[10] N. Brambilla, J. Ghiglieri, A. Vairo, and P. Petreczky, Static quark-antiquark pairs at finite temperature, Phys. Rev. D 78, 014017 (2008).

[11] N. Brambilla, M. A. Escobedo, J. Ghiglieri, J. Soto, and A. Vairo, Heavy quarkonium in a weakly coupled quark-gluon plasma below the melting temperature, J. High Energy Phys. 09 (2010) 038.

[12] A. Vairo, Quarkonium in a weakly coupled quark-gluon plasma, AIP Conf. Proc. 1317, 241 (2011).

[13] N. Brambilla, M. A. Escobedo, J. Ghiglieri, and A. Vairo, Thermal width and gluodissociation of quarkonium in pNRQCD, J. High Energy Phys. 12 (2011) 116.

[14] N. Brambilla, M. A. Escobedo, J. Ghiglieri, and A. Vairo, Thermal width and quarkonium dissociation by inelastic parton scattering, J. High Energy Phys. 05 (2013) 130.

[15] M. A. Escobedo, F. Giannuzzi, M. Mannarelli, and J. Soto, Heavy quarkonium moving in a quark-gluon plasma, Phys. Rev. D 87, 114005 (2013).

[16] B. Krouppa and M. Strickland, Predictions for bottomonia suppression in 5.023 TeV Pb-Pb collisions, Universe 2, 16 (2016).

[17] B. Krouppa, R. Ryblewski, and M. Strickland, Bottomonia suppression in 2.76 TeV Pb-Pb collisions, Phys. Rev. C 92, 061901 (2015).

[18] M. Strickland and D. Bazow, Thermal bottomonium suppression at RHIC and LHC, Nucl. Phys. A879, 25 (2012).

[19] S. Chatrchyan et al. (CMS Collaboration), Observation of Sequential Upsilon Suppression in PbPb Collisions, Phys. Rev. Lett. 109, 222301 (2012).

[20] G. G. Fronzé(ALICECollaboration), $\Upsilon$ production in $\mathrm{p}-\mathrm{Pb}$ and $\mathrm{Pb}-\mathrm{Pb}$ collisions with ALICE at the LHC, arXiv:1612.06691.

[21] L. Adamczyk et al. (STAR Collaboration), Upsilon production in $\mathrm{U}+\mathrm{U}$ collisions at $193 \mathrm{GeV}$ with the STAR experiment, Phys. Rev. C 94, 064904 (2016).

[22] A. Pineda and J. Soto, Effective field theory for ultrasoft momenta in NRQCD and NRQED, Nucl. Phys. B, Proc. Suppl. 64, 428 (1998).

[23] N. Brambilla, A. Pineda, J. Soto, and A. Vairo, Potential NRQCD: An effective theory for heavy quarkonium, Nucl. Phys. B566, 275 (2000).

[24] N. Brambilla, A. Pineda, J. Soto, and A. Vairo, Effective field theories for heavy quarkonium, Rev. Mod. Phys. 77, 1423 (2005).
[25] J. Casalderrey-Solana and D. Teaney, Heavy quark diffusion in strongly coupled $N=4$ Yang-Mills, Phys. Rev. D 74, 085012 (2006).

[26] S. Caron-Huot and G. D. Moore, Heavy Quark Diffusion in Perturbative QCD at Next-to-Leading Order, Phys. Rev. Lett. 100, 052301 (2008).

[27] A. Francis, O. Kaczmarek, M. Laine, T. Neuhaus, and H. Ohno, Nonperturbative estimate of the heavy quark momentum diffusion coefficient, Phys. Rev. D 92, 116003 (2015).

[28] S. Borsányi, Z. Fodor, C. Hoelbling, S. D. Katz, S. Krieg, C. Ratti, and K. K. Szabó, Is there still any $T_{c}$ mystery in lattice QCD? Results with physical masses in the continuum limit III, J. High Energy Phys. 09 (2010) 073.

[29] A. Bazavov et al. (HotQCD Collaboration), Equation of state in (2+ 1)-flavor QCD, Phys. Rev. D 90, 094503 (2014).

[30] A. Bazavov, N. Brambilla, H.-T. Ding, P. Petreczky, H.-P. Schadler, A. Vairo, and J. H. Weber, Polyakov loop in $2+1$ flavor QCD from low to high temperatures, Phys. Rev. D 93, 114502 (2016).

[31] D. Banerjee, S. Datta, R. Gavai, and P. Majumdar, Heavy quark momentum diffusion coefficient from lattice QCD, Phys. Rev. D 85, 014510 (2012).

[32] N. Brambilla, M. A. Escobedo, J. Soto, and A. Vairo, Report No. TUM-EFT 89/16 (unpublished).

[33] J. Berges, Introduction to nonequilibrium quantum field theory, AIP Conf. Proc. 739, 3 (2005).

[34] G. Lindblad, On the generators of quantum dynamical semigroups, Commun. Math. Phys. 48, 119 (1976).

[35] V. Gorini, A. Kossakowski, and E. C. G. Sudarshan, Completely positive dynamical semigroups of $\mathrm{N}$ level systems, J. Math. Phys. (N.Y.) 17, 821 (1976).

[36] Y. Akamatsu, Heavy quark master equations in the Lindblad form at high temperatures, Phys. Rev. D 91, 056002 (2015).

[37] J. D. Bjorken, Highly relativistic nucleus-nucleus collisions: The central rapidity region, Phys. Rev. D 27, 140 (1983).

[38] W. M. Alberico, A. Beraudo, A. De Pace, A. Molinari, M. Monteno, M. Nardi, F. Prino, and M. Sitta, Heavy flavors in $A A$ collisions: Production, transport and final spectra, Eur. Phys. J. C 73, 2481 (2013).

[39] S. Chatrchyan et al. (CMS Collaboration), Observation and studies of jet quenching in $\mathrm{PbPb}$ collisions at nucleonnucleon center-of-mass energy $=2.76 \mathrm{TeV}$, Phys. Rev. C 84, 024906 (2011).

[40] P. L. Cho and A. K. Leibovich, Color octet quarkonia production. 2., Phys. Rev. D 53, 6203 (1996).

[41] V. Khachatryan et al. (CMS Collaboration), Suppression of $\Upsilon(1 S), \Upsilon(2 S)$ and $\Upsilon(3 S)$ production in $\mathrm{PbPb}$ collisions at $\sqrt{s_{N N}}=2.76 \mathrm{TeV}$, Phys. Lett. B 770, 357 (2017).

[42] X. Du, R. Rapp, and M. He, Color screening and regeneration of bottomonia at RHIC and the LHC, arXiv:1706.08670.

[43] Y. Akamatsu and A. Rothkopf, Stochastic potential and quantum decoherence of heavy quarkonium in the quarkgluon plasma, Phys. Rev. D 85, 105011 (2012).

[44] R. Katz and P. B. Gossiaux, The Schrödinger-Langevin equation with and without thermal fluctuations, Ann. Phys. (Amsterdam) 368, 267 (2016).

[45] F. Gelis, Color glass condensate and Glasma, Int. J. Mod. Phys. A 28, 1330001 (2013).

[46] http:www.universe-cluster.de. 